\title{
Birth of discrete Lorenz attractors at the bifurcations of 3D maps with homoclinic tangencies to saddle points.
}

\author{
S.V.Gonchenko ${ }^{1}$, I.I.Ovsyannikov ${ }^{2}$ and J.C.Tatjer ${ }^{3}$ \\ ${ }^{1}$ Nizhny Novgorod State University, Russia; \\ E-mail: gonchenko@pochta.ru \\ ${ }^{2}$ Universität Bremen, Fachbereich 3, Bibliotekstrasse 1, 28359 Bremen, Germany \\ Nizhny Novgorod State University, Russia; \\ E-mail: ivan.i.ovsyannikov@gmail.com \\ ${ }^{3}$ Dept. de Matemàtica Aplicada i Anàlisi, Universitat de Barcelona, Spain; \\ E-mail: jcarles@maia.ub.es
}

\begin{abstract}
It was established in [1] that bifurcations of three-dimensional diffeomorphisms with a homoclinic tangency to a saddle-focus fixed point with the Jacobian equal to 1 can lead to Lorenz-like strange attractors. In the present paper we prove an analogous result for three-dimensional diffeomorphisms with a homoclinic tangency to a saddle fixed point with the Jacobian equal to 1, provided the quadratic homoclinic tangency under consideration is non-simple.

Keywords: Homoclinic tangency, rescaling, 3D Hénon map, bifurcation, Lorenz-like attractor.

Mathematics Subject Classification: 37C05, 37G25, 37G35
\end{abstract}

\section{Introduction}

In this paper we describe a new class of homoclinic tangencies whose bifurcations lead to the birth of strange attractors. We call the attractor that appears in the Poincare map of a periodically perturbed flow with a Lorenz attractor a discrete Lorenz attractor. A theory of such attractors was built in [2]. They can emerge in a wide class of maps which do not need to be directly linked to periodically perturbed flows. Thus, a discrete Lorenz attractor was first found in [3] for the three-dimensional Hénon map

$$
\bar{x}=y, \quad \bar{y}=z, \quad \bar{z}=M_{1}+B x+M_{2} y-z^{2},
$$

where it exists for some open domain of the parameters $\left(M_{1}, M_{2}, B\right)$ adjoining to the point $\left(M_{1}=-1 / 4, B=1, M_{2}=1\right)$ where the map has a fixed point with the triplet of multipliers $(-1,-1,+1)$. It is shown in [4 that the normal form for the bifurcations of such fixed point is the Shimizu-Morioka model subject to exponentially small periodic perturbation; 
the Shimizu-Morioka model has a region of parameter values which corresponds to the geometrical Lorenz attractor [5, 6], therefore it is quite typical for a map undergoing the bifurcation of $(-1,-1,+1)$ to have a discrete Lorenz attractor. Indeed, these attractors were further found numerically in several types of generalized 3D Hénon maps [7], and in models of non-holonomic mechanics such as Celtic stone [8], see also [9, 10]. Simple universal bifurcation scenarios that lead to a discrete Lorenz attractor are described in [11, 12 .

Discrete Lorenz attractors belong to the class of the so-called wild hyperbolic attractors [13] which admit homoclinic tangencies and, hence, contain wild hyperbolic sets [14], however, bifurcations of these tangencies do not lead to the birth of periodic sinks. The main reason of this for discrete Lorenz attractors is that they possess a pseudo-hyperbolic structure. This, very briefly, means that the differential $D f$ of the corresponding map $f$, in the restriction onto an absorbing neighbourhood $\mathcal{D}$ of the attractor, admits an invariant splitting of the form $E_{x}^{s s} \oplus E_{x}^{u c}$, depending continuously on the point $x \in \mathcal{D}$, such that $D f$ is strongly contracting in restriction to $E^{s s}$ and expands volume in $E^{u c}$ (see [13, 2] for more detail). This property is robust and prevents from the existence of stable periodic orbits, therefore the discrete Lorenz attractors preserve "strangeness" at small smooth perturbations. This distinguishes them from numerous "physical" attractors (quasiattractors in the terminology by Afraimovich and Shilnikov [15]), such as Hénon-like attractors, spiral and screw attractors (Rössler attractors, attractors in the Chua circuits) etc., in which periodic sinks (of arbitrary large periods) can appear under arbitrary small perturbations.

The fact that strange (e.g. Lorenz-like) attractors can appear at the bifurcations of homoclinic tangencies in multidimensional case was announced yet in [16]. In [1] it was shown that the discrete Lorenz-like attractors appear at the bifurcations of threedimensional diffeomorphisms with a homoclinic tangency to a saddle-focus fixed point with the Jacobian equal to 1. Analogous results were obtained in [17, 18, 19] for the bifurcations of three-dimensional diffeomorphisms with a nontransversal heteroclinic cycle containing two fixed points, one with the Jacobian less than 1 and the other with the Jacobin greater than 1.

Note that in all these papers it was assumed that at least one of the fixed points is a saddle-focus (i.e. it has a pair of complex conjugate multipliers inside the unit circle and one real multiplier outside). This, along with the conditions on the Jacobians of the fixed points, means that the so-called effective dimension $d_{e}$ of the problem (see [20]) is equal to 3 , which is necessary as the discrete Lorenz-like attractors can exist only in three- and higher-dimensional diffeomorphisms.

In the present paper we study the case of a quadratic homoclinic tangency to a fixed point $O$ of saddle type, i.e. we assume that all three multipliers of the fixed point are real and different. Then, as it is known from [16, 20, 21], to have the effective dimension of 
the corresponding problem equal to 3 , we need to assume that (i) the Jacobian $J$ at the fixed point is equal to \pm 1 and (ii) the quadratic homoclinic tangency is non-simple (see Definition 1 in section 1).

The first studies of a codimension-two non-simple homoclinic tangency were performed in [21] where it was called a generalized homoclinic tangency. The notion of a simple quadratic homoclinic tangency (a variant of the so-called quasitransversal homoclinic intersection [22]) was introduced in [16]. For three-dimensional maps with a homoclinic tangency to a saddle fixed point $O$ with multipliers $\nu_{i}, i=1,2,3$ such that $\left|\nu_{1}\right|<\left|\nu_{2}\right|<\left|\nu_{3}\right|$, the simplicity implies the existence of a non-local two-dimensional invariant manifold, for the map itself and for all $C^{1}$-close maps. This manifold contains all orbits entirely lying in a small fixed neighbourhood of the homoclinic orbit. If the point $O$ has type $(2,1)$, i.e. $\left|\nu_{1,2}\right|<1<\left|\nu_{3}\right|$, this manifold is attractive; if the point $O$ has type $(1,2)$, i.e. $\left|\nu_{1}\right|<1<\left|\nu_{2,3}\right|$, the manifold is repelling. It follows that neither periodic nor strange attractors can be born at the bifurcation of a simple tangency if $\left|\nu_{2} \nu_{3}\right|>1$. However, as it was shown in [21, if the tangency is non-simple, then periodic attractors can appear provided $|J|=\left|\nu_{1} \nu_{2} \nu_{3}\right|<1$, see also [23] where the case of a saddle point of type $(2,1)$ was considered in more detail. These results are important for the theory of dynamical chaos since they show that the non-simple homoclinic tangencies can destroy "strangeness" of attractors as they may lead to the birth of periodic sinks.

Let $f_{0}$ be a three-dimensional orientable $C^{r}$-diffeomorphism, $r \geq 3$, satisfying the following conditions:

A) $f_{0}$ has a saddle fixed point $O$ with multipliers $\lambda_{1}, \lambda_{2}, \gamma$ such that $0<\left|\lambda_{2}\right|<\left|\lambda_{1}\right|<$ $1<|\gamma|$ (a saddle of type $(2,1))$;

B) The Jacobian $J_{1} \equiv \lambda_{1} \lambda_{2} \gamma$ of $f_{0}$ at the fixed point $O$ is equal to 1 (a saddle of conservative type);

C) the unstable manifold $W^{u}(O)$ has a quadratic tangency with $W^{s}(O)$ at the points of some homoclinic orbit $\Gamma_{0}$;

D) the tangency is non-simple (see Definition 1) and nondegenerate.

Diffeomorphisms close to $f_{0}$ and satisfying conditions $\mathrm{A}-\mathrm{D}$ compose, in the space of $C^{r}$-diffeomorphisms, a locally connected bifurcation surface of codimension 3 . Thus, in order to study bifurcations of $f_{0}$, we need to consider, first of all, three-parameter generic unfoldings; the parameters must control the unfolding of degeneracies given by the conditions $\mathrm{B}, \mathrm{C}$, and $\mathrm{D}$. Let $f_{\mu}$ be such a family, where $\mu=\left(\mu_{1}, \mu_{2}, \mu_{3}\right)$. We choose the parameters as follows: $\mu_{1}$ is the splitting parameter (which controls condition $\mathrm{C}$ ); $\mu_{2}$ controls condition D) in such a way that, at $\mu_{1}=0$, the tangency becomes simple for $\mu_{2} \neq 0$; and $\mu_{3}$ controls the Jacobian at $O$, i.e it can be taken equal e.g. to $\mu_{3}=1-\lambda_{1} \lambda_{2} \gamma$. The main result of the present paper is the following 
Main Theorem. Let $f_{\mu}$ be the three-parametric family under consideration $\left(f_{0}\right.$ satisfies $A-D$ and $f_{\mu}$ unfolds the degeneracies given by conditions $B, C$ and $D$ in a generic way). Then, in any neighbourhood of the origin $\mu=0$ in the parameter space there exist infinitely many domains $\delta_{k}$, where $\delta_{k} \rightarrow(0,0,0)$ as $k \rightarrow \infty$, such that the diffeomorphism $f_{\mu}$ has a discrete Lorenz-like attractor at $\mu \in \delta_{k}$.

The method of the proof is as follows. For the diffeomorphisms $f_{\mu}$ we construct firstreturn maps $T_{k}(\mu)$ defined in some neighbourhoods $\sigma_{0}^{k}$ near a point of orbit $\Gamma_{0}$; there are infinitely many such $\sigma_{0}^{k}$ depending on the return time $k, k=\bar{k}, \bar{k}+1, \ldots$ Further, we rescale coordinates and parameters and show that, for every sufficiently large $k$, there is an open domain $\Delta_{k}$ of values of $\mu$ such that (i) $\Delta_{k} \rightarrow(0,0,0)$ as $k \rightarrow \infty$ and (ii) the first return map $T_{k}(\mu)$ for $\mu \in \Delta_{k}$, in the rescaled coordinates $(x, y, z)$ and parameters $\left(M_{1}, M_{2}, B\right)$, takes the form (0.1) up to terms that are asymptotically small as $k \rightarrow \infty$. It is important to note that the rescaled coordinates and parameters can take arbitrary finite values (all positive values for $B$ ) as $k$ grows. Thus, we can apply the results from [1, 3] about the existence of discrete Lorenz-like attractors in the 3D Hénon map (0.1) and, hence, deduce the existence of such attractor for the map $T_{k}(\mu)$ for $\mu \in \delta_{k} \subset \Delta_{k}$.

In fact, our analysis provides useful results on global bifurcations in another interesting case of a non-simple homoclinic tangency. Namely, consider an orientable diffeomorphism $g_{0}$ which has a saddle fixed point with real multipliers $\lambda, \gamma_{1}, \gamma_{2}$ such that $0<|\lambda|<1<$ $\left|\gamma_{1}\right|<\left|\gamma_{2}\right|$ and $\lambda \gamma_{1} \gamma_{2}=1$ (a conservative saddle of type $(1,2)$ ). Suppose also that $g_{0}$ has a quadratic non-simple tangency at the points of some homoclinic orbit $\hat{\Gamma}_{0}$. Then we can assume that $g_{0}=f_{0}^{-1}$ and, thus, one can obviously use bifurcation results obtained for $f_{0}$. However, there is an essential difference in the interpretation of results. Namely, the Main Theorem gives, for $g_{0}$, only the existence of discrete Lorenz-like repellers, not attractors.

As $g_{\mu}$ is inverse to $f_{\mu}$, the first-return maps $\hat{T}_{k}$ for $g_{\mu}$ are inverse to the first-return maps $T_{k}$ for $f_{\mu}$ and, thus, in the corresponding parameter domains, the rescaled map $\hat{T}_{k}$ is close to the inverse of (0.1), i.e. to the map

$$
\bar{x}=y, \quad \bar{y}=z, \quad \bar{z}=\hat{M}_{1}+\hat{M}_{2} z+\hat{B} x-y^{2},
$$

where $\hat{B}=B^{-1}, \hat{M}_{1}=\frac{M_{1}}{B^{2}}, \hat{M}_{2}=-\frac{M_{2}}{B}$.

Map (0.2) is well-known in homoclinic dynamics, see e.g. [16, 21, 24, 25]. When $\hat{B}=0$ map (0.2) becomes two-dimensional: the variable $x$ decouples and we have (for the coordinates $y$ and $z$ ) the map of the following form: $\bar{y}=z, \bar{z}=\hat{M}_{1}+\hat{M}_{2} z-y^{2}$. This map is called Mira map; its dynamics was extensively studied, see e.g. [26]. In particular, at certain parameter values this non-invertible two-dimensional map may have a strange attractor with two positive Lyapunov exponents [27, 28]. Numerical experiments in this case suggest that the non-robust strange attractors that have the sum of their Lyapunov 
exponents positive form a set of large measure in the parameter plane. Numerics also shows that this property, to have two positive Lyapunov exponents, is inherited by the map (0.2) with $B \neq 0$. The chaotic dynamics of map (0.2) was studied e.g. in [11] where it was shown (using the results from [29]) that map (0.2) possesses strange attractors (quasiattractors) of spiral type, i.e. those containing a saddle-focus fixed point with two-dimensional unstable manifold. The question of the existence of genuine strange attractors (e.g. discrete Lorenz attractors), is open for map (0.2), though this problem is very interesting.

The contents of the paper is as follows. Section 1 contains the statement of the problem and all necessary definitions including the definition of two types of non-simple homoclinic tangencies. In Section 2 we construct the first return maps $T_{k}$ and formulate the main technical result, Rescaling Lemma 1. Proof of Lemma 1 is given in Section 3.

\section{Statement of the problem and main definitions}

Let $f_{0}$ be a three-dimensional $C^{r}$-diffeomorphism, $r \geq 3$, satisfying the conditions A)-D). We embed $f_{0}$ into a three parameter family $f_{\mu}$ (general unfolding under conditions $\mathrm{B}$ )D)) with the parameters $\left(\mu_{1}, \mu_{2}, \mu_{3}\right)$ described as above. We choose a sufficiently small fixed neighbourhood $U \equiv U\left(O \cup \Gamma_{0}\right)$ of the orbit $\Gamma_{0}$. Note that $U$ is a union of a ball $U_{0}$ containing the point $O$ and a number of balls surrounding those points of $\Gamma_{0}$ which lie outside $U_{0}$.

Denote by $T_{0}(\mu)$ the restriction of the diffeomorphism $f_{\mu}$ onto $U_{0}$. The map $T_{0}=\left.f_{\mu}\right|_{U_{0}}$ is called a local map. It is known, [25, 30, 31, 32, that $T_{0}(\mu)$ can be represented in some $C^{r}$-smooth local coordinates $\left(x_{1}, x_{2}, y\right)$ from $U_{0}$, smoothly $\left(C^{r-2}\right)$ depending on $\mu$, in the following main normal form:

$$
\begin{aligned}
& \bar{x}_{1}=\lambda_{1}(\mu) x_{1}+\tilde{H}_{1}(y, \mu) x_{2}+O\left(\|x\|^{2}|y|\right) \\
& \bar{x}_{2}=\lambda_{2}(\mu) x_{2}+\tilde{R}_{2}(x, \mu)+\tilde{H}_{2}(y, \mu) x_{2}+O\left(\|x\|^{2}|y|\right) \\
& \bar{y}=\gamma(\mu) y+O\left(\|x\||y|^{2}\right)
\end{aligned}
$$

where $\tilde{H}_{1,2}(0, \mu)=0, \tilde{R}_{2}(x, \mu)=O\left(\|x\|^{2}\right)$.

We note that, in these coordinates, the local invariant manifolds, stable $W_{l o c}^{s}$, unstable $W_{l o c}^{u}$ and strong stable $W_{l o c}^{s s}$, of the point $O$ are all straightened in $U_{0}$ : their equations are as follows $W_{l o c}^{s}(O):\{y=0\}, W_{l o c}^{u}(O):\left\{x_{1}=0, x_{2}=0\right\}$ and $W_{l o c}^{s s}(O):\left\{x_{1}=0, y=0\right\}$.

The intersection points of $\Gamma_{0}$ with $U_{0}$ belong to the set $W^{s} \cap W^{u}$ and accumulate at $O$. Thus, infinitely many points of $\Gamma_{0}$ lie on $W_{l o c}^{s}$ and $W_{l o c}^{u}$. Let $M^{+}\left(x_{1}^{+}, x_{2}^{+}, 0\right) \in W_{l o c}^{s}$ and $M^{-}\left(0,0, y^{-}\right) \in W_{l o c}^{u}$ be two of such points and let $M^{+}=f_{0}^{n_{0}}\left(M^{-}\right)$for some positive integer $n_{0}$. Let $\Pi^{+} \subset U_{0}$ and $\Pi^{-} \subset U_{0}$ be small enough neighbourhoods of the points $M^{+}$ and $M^{-}$, respectively. The map $T_{1}(\mu) \equiv f_{\mu}^{n_{0}}: \Pi^{-} \rightarrow \Pi^{+}$is called a global map. 


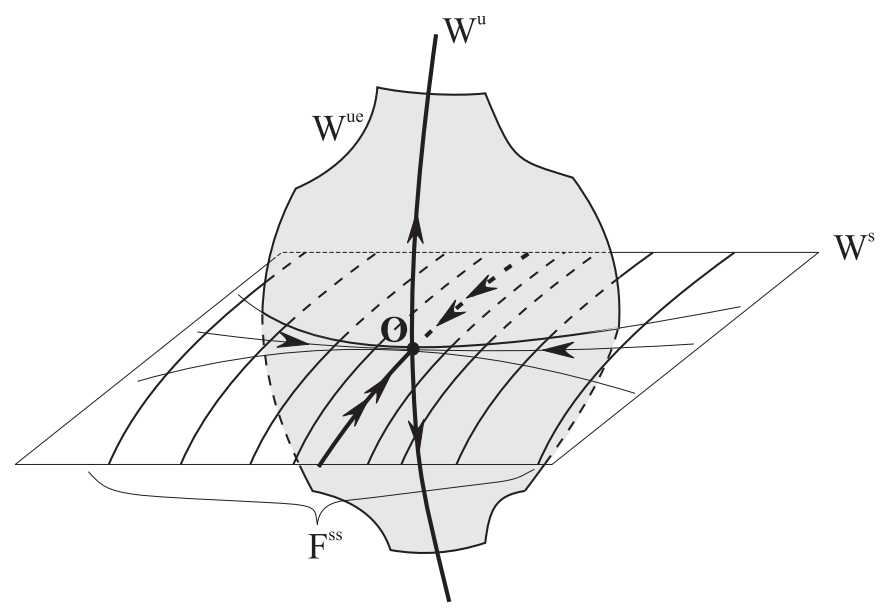

Figure 1: A part of the strong stable foliation $F^{s s}$ containing the strong stable manifold $W^{s s}$; and a piece of one of the extended unstable manifolds $W^{u e}$ containing $W^{u}$ and being transversal to $W^{s s}$ at $O$.

In order to formulate explicitly conditions $\mathrm{C}$ and $\mathrm{D}$ on the homoclinic tangency we recall first some important facts from the theory of invariant manifolds [30, 33].

When the condition $\mathrm{A}$ is fulfilled, in $U_{0}$ there exist the so-called extended unstable manifolds $W^{u e}(O)$, see Fig. 1 where an example of such a manifold is presented. There are infinitely many (continuum) such manifolds, they are only $C^{1+\varepsilon}$-smooth in general. In the case under consideration, all $W^{u e}(O)$ are two-dimensional, contain $W^{u}$ and touch at the point $O$ the eigendirection of $D f_{0}$ corresponding to the multiplier $\lambda_{1}$ - the line $\left\{y=0, x_{2}=0\right\}$ in coordinates (1.1). Thus, each $W_{\text {loc }}^{u e}(O)$ has the equation of form $x_{2}=\varphi\left(x_{1}, y\right)$, where $\varphi(0, y) \equiv 0$ and $\varphi_{x_{1}}^{\prime}(0,0)=0$. Note that in coordinates (1.1) all the manifolds $W^{u e}(O)$ have the same tangent plane $\left\{x_{2}=0\right\}$ at each point of $W^{u}(O)$.

Another fact we use is the existence of a strong stable invariant foliation, see Fig. 1. Recall that $W^{s}(O)$ contains the one-dimensional strong stable submanifold $W^{s s}(O)$, which is invariant, $C^{r}$-smooth and touches at $O$ the eigenvector corresponding to the strong stable (nonleading) multiplier $\lambda_{2}$. Moreover, the manifold $W^{s}(O)$ is foliated near $O$ by the foliation $F^{s s}$ which is $C^{r}$-smooth, unique and contains $W^{s s}$ as a leaf. As we said before, in coordinates (1.1), $W_{\text {loc }}^{s s}(O)$ has the equation $\left\{x_{1}=0, y=0\right\}$ and also the foliation $F^{s s}$ on $U_{0}$ consists of the leaves $\left\{x_{1}=\right.$ const, $\left.y=0\right\}$.

Denote the tangent plane to $W^{u e}(O)$ at the point $M^{-}$as $P^{u e}\left(M^{-}\right)$. By our condition, the curve $T_{1}\left(W_{l o c}^{u} \cap \Pi^{-}\right)$has at $\mu=0$ a quadratic tangency with $W_{l o c}^{s}$ at the point $M^{+}$.

Definition 1 The homoclinic tangency under consideration is called simple if $T_{1}\left(P^{u e}\left(M^{-}\right)\right)$ intersects transversely the leaf $F^{s s}\left(M^{+}\right)$of the foliation $F^{s s}$ containing the point $M^{+}$. If this condition is not fulfilled we call such quadratic tangency non-simple. 


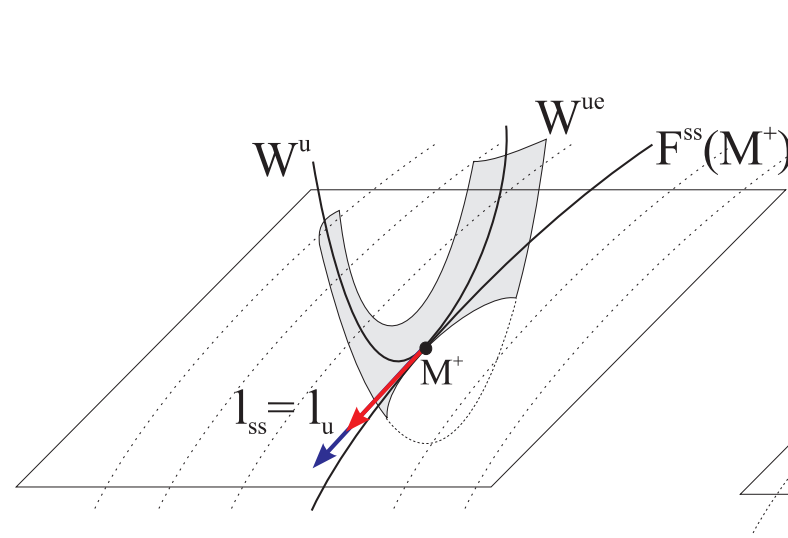

a)

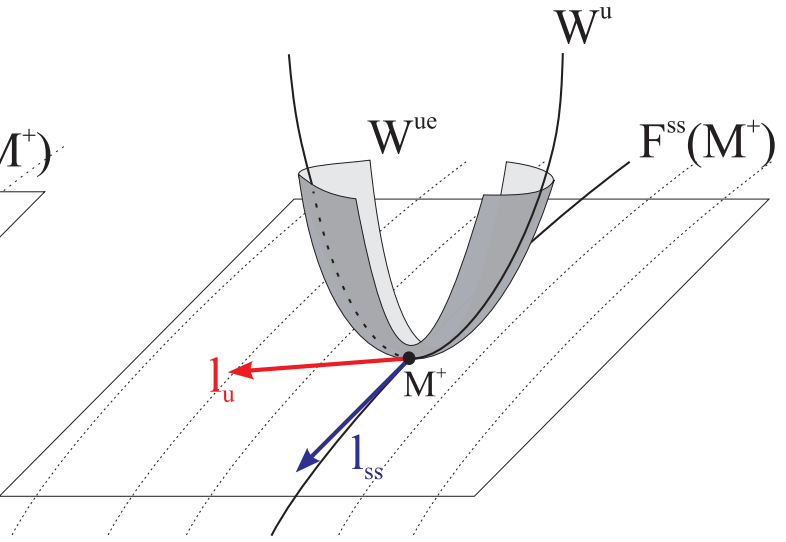

b)

Figure 2: Two types of nondegenerate non-simple quadratic (homoclinic) tangency: (a) $W^{u e}$ is transversal to $W_{l o c}^{s}$ and touches the leaf $F^{s s}\left(M^{+}\right)$; (b) $W^{u e}$ is tangent to $W_{l o c}^{s}$ and the curves $W^{u}$ and $F^{s s}\left(M^{+}\right)$ has a general intersection at $M^{+}$.

According to [21] we define two general cases of non-simple homoclinic tangencies:

Case I. The surface $T_{1}\left(P^{u e}\left(M^{-}\right)\right)$is transversal to the plane $W_{\text {loc }}^{s}(O)$ but is tangent to the line $F^{s s}\left(M^{+}\right)$at $M^{+}$.

Case II. The surfaces $T_{1}\left(P^{u e}\left(M^{-}\right)\right)$and $W_{\text {loc }}^{\text {s }}(O)$ have a tangency at $M_{1}^{+}$and the curves $T_{1}\left(W_{\text {loc }}^{u}(O) \cap \Pi^{-}\right)$and $F^{s s}\left(M^{+}\right)$have a general intersection.

Thus, in Case I the tangent vectors $l_{u}$ to $T_{1}\left(W_{l o c}^{u}\right)$ and $l_{s s}$ to $F_{1}^{s s}\left(M_{1}^{+}\right)$are collinear, while in Case II these vectors have different directions, see Fig. 2 (a) and (b).

\section{Calculation of the first return maps $T_{k}(\mu)$.}

We consider on $U_{0}$ the local coordinates $\left(x_{1}, x_{2}, y\right)$ in which the map $T_{0}(\mu)$ has the form (1.1). Let $\left\{p_{i}\left(x_{i 1}, x_{i 2}, y_{i}\right)\right\}, i=1,2, \ldots, k$, be such points in $U_{0}$ that $p_{i+1}=T_{0}\left(p_{i}\right)$. Then, by [25, 30, 32], we can represent the map $T_{0}^{k}: U_{0} \rightarrow U_{0}$ in the so-called Shilnikov crossform as follows.

$$
\begin{aligned}
& x_{k 1}-\lambda_{1}^{k}(\mu) x_{01}=\hat{\lambda}^{k} \xi_{k 1}\left(x_{0}, y_{k}, \mu\right) \\
& x_{k 2}-\lambda_{2}^{k}(\mu) x_{02}=\hat{\lambda}^{k} \xi_{k 2}\left(x_{0}, y_{k}, \mu\right) \\
& y_{0}-\gamma(\mu)^{-k} y_{k}=\hat{\gamma}^{-k} \eta_{k}\left(x_{0}, y_{k}, \mu\right),
\end{aligned}
$$

where $\hat{\lambda}$ and $\hat{\gamma}$ are some constants such that $0<\hat{\lambda}<\left|\lambda_{1}(\mu)\right|, \hat{\gamma}>|\gamma(\mu)|$ and functions $\xi_{k}$ and $\eta_{k}$ are uniformly bounded along with all derivatives up to order $(r-2)$. Moreover, 
$\left\|x_{k}\right\|_{C^{r-1}}=O\left(\lambda_{1}^{k}\right),\left\|y_{0}\right\|_{C^{r-1}}=O\left(\gamma^{-k}\right),\left\|x_{k}, y_{0}\right\|_{C^{r}} \rightarrow 0$ as $k \rightarrow \infty$, see [23] for more details.

To construct the global map $T_{1}$ we use the facts that when $\mu=0$ we have $T_{1}\left(M^{-}\right)=$ $M^{+}$, where, recall, $M^{+}=\left(x_{1}^{+}, x_{2}^{+}, 0\right)$ and $M^{-}=\left(0,0, y^{-}\right)$is a pair of points of $\Gamma_{0}$, and $T_{1}\left(W_{l o c}^{u}\right)$ and $W_{l o c}^{s}$ have a quadratic tangency at the point $M^{+}$. Accordingly, the global map $T_{1}$ at all small $\mu$ can be written as

$$
\begin{aligned}
& \bar{x}_{1}-x_{1}^{+}=a_{11} x_{1}+a_{12} x_{2}+b_{1}\left(y-y^{-}\right)+O\left(\|x\|^{2}+\left(y-y^{-}\right)^{2}\right) \\
& \bar{x}_{2}-x_{2}^{+}=a_{21} x_{1}+a_{22} x_{2}+b_{2}\left(y-y^{-}\right)+O\left(\|x\|^{2}+\left(y-y^{-}\right)^{2}\right) \\
& \bar{y}=y^{+}(\mu)+c_{1} x_{1}+c_{2} x_{2}+d\left(y-y^{-}\right)^{2}+O\left(\|x\|^{2}+\|x\| y-y^{-}|+| y-\left.y^{-}\right|^{3}\right)
\end{aligned}
$$

where $y^{+}(0)=0$ and coefficients $a_{11}, \ldots, d$ as well as $x^{+}$and $y^{-}$depend smoothly on $\mu$. Note that since the homoclinic tangency at $\mu=0$ is quadratic, we have $d \neq 0$. Moreover, map $T_{1}(0)$ is a diffeomorphism, therefore

$$
J_{1}=\operatorname{det}\left(\begin{array}{ccc}
a_{11} & a_{12} & b_{1} \\
a_{21} & a_{22} & b_{2} \\
c_{1} & c_{2} & 0
\end{array}\right) \neq 0
$$

and, hence, $b_{1}^{2}+b_{2}^{2} \neq 0, c_{1}^{2}+c_{2}^{2} \neq 0$ at $\mu=0$.

Now we consider condition D separately for Cases I and II.

Case I. The tangent plane $P^{u e}\left(M^{-}\right)$to $W_{l o c}^{\text {ue }}$ at point $M^{-}$has equation $x_{2}=0$. The equation of $T_{1}\left(P_{u e}\left(M^{-}\right)\right)$at $\mu=0$ is obtained by putting $x_{2}=0$ into (2.2). Then the transversality of $T_{1}\left(P^{u e}\left(M^{-}\right)\right)$and $W_{l o c}^{s}(\bar{y}=0)$ yields $c_{1}(0) \neq 0$. The tangent vector to the line $T_{1}\left(P_{u e}\left(M^{-}\right)\right) \cap W_{\text {loc }}^{s}$ at point $M^{+}$is $\left(b_{1}(0), b_{2}(0), 0\right)$. The equation of the leaf $F^{s s}\left(M^{+}\right)$is $\left\{x_{1}=x_{1}^{+}, y=0\right\}$. Therefore, the tangency of $T_{1}\left(P^{u e}\left(M^{-}\right)\right)$and $F^{s s}\left(M^{+}\right)$ implies $b_{1}(0)=0$. In this case $b_{2} \neq 0$ and $a_{11}^{2}+a_{12}^{2} \neq 0$ because of (2.3). Thus, in Case I map $T_{1}(\mu)$ has form (2.2) where

$$
b_{1}(0)=0, c_{1}(0) \neq 0 \text { and } b_{2}(0) \neq 0 .
$$

Case II. The equation of $T_{1}\left(P^{u e}\left(M^{-}\right)\right)$at $\mu=0$ is the same as in Case I. Then the tangency of $T_{1}\left(P^{u e}\left(M^{-}\right)\right)$and $W_{l o c}^{s}$ at $\mu=0$ implies that $c_{1}(0)=0$. Also, the tangent vectors to the lines $T_{1}\left(L_{u}\right)$ and to $F^{s s}\left(M^{+}\right)$at point $M^{+}$are non-parallel if $b_{1}(0) \neq 0$. Thus, in Case II map $T_{1}(\mu)$ has form (2.2) where

$$
c_{1}(0)=0, b_{1}(0) \neq 0 \text { and } c_{2}(0) \neq 0 .
$$

The main goal of the paper is to study bifurcations of single-round periodic orbits of diffeomorphisms close to $f_{0}$. Every point of such an orbit can be considered as a fixed point for the corresponding first return map $T_{k}$, where $k$ can run all sufficiently large integers. These maps are constructed as the composition $T_{k}=T_{1} T_{0}^{k}: \Pi^{+} \rightarrow \Pi^{-} \rightarrow \Pi^{+}$, 
where, recall, $\Pi^{+} \subset U_{0}$ and $\Pi^{-} \subset U_{0}$ are small neighbourhoods of the homoclinic points $M^{+}$and $M^{-}$, respectively. If we take a point $M \in \Pi^{+}$, then its iterations under the local map $T_{0}$ can reach $\Pi^{-}$. Such points form on $\Pi^{+}$a set consisting of infinitely many threedimensional strips $\sigma_{k}^{0}=T_{0}^{-k}\left(\Pi^{-}\right) \cap \Pi^{+}, k \in\left\{k_{0}, k_{0}+1, \ldots\right\}$. Accordingly, $\sigma_{k}^{1}=T_{0}^{k}\left(\sigma_{k}^{0}\right)$ is a (three-dimensional) strip on $\Pi^{-}$. Strips $\sigma_{k}^{0}$ and $\sigma_{k}^{1}$ accumulate on $W_{l o c}^{s} \cap \Pi^{+}$and $W_{l o c}^{u} \cap \Pi^{-}$, respectively, as $k \rightarrow \infty$. The global map $T_{1}$ maps the strip $\sigma_{k}^{1}$ into a threedimensional horseshoe $T_{1}\left(\sigma_{k}^{1}\right) \subset \Pi^{+}$. By the definition, map $T_{k}=T_{1} T_{0}^{k}: \sigma_{k}^{0} \rightarrow \Pi^{+}, k \in$ $\left\{k_{0}, k_{0}+1, \ldots\right\}$, is the first return map. Since $T_{1}$ is the $n_{0}^{\text {th }}$ power of $f_{\mu}$, any fixed point of $T_{k}$ corresponds to a single-round periodic orbit of $f_{\mu}$ of period $k+n_{0}$.

We consider three-parameter families $f_{\mu_{1}, \mu_{2}, \mu_{3}}$ of diffeomorphisms close to $f$. Naturally, a parameter $\mu_{1}$ of the splitting of manifolds $W^{s}(O)$ and $W^{u}(O)$ with respect to the point $M^{+}$is considered as one of the governing parameters. It is seen from (2.2) that

$$
\mu_{1} \equiv y^{+}(\mu)
$$

As the second parameter, we consider a parameter $\mu_{2}$ resolving the degeneracy connected either with condition $\mathrm{D}_{\mathrm{I}}$ (in Case I) or with condition $\mathrm{D}_{\mathrm{II}}$ (in Case II). It is convenient to take directly

$$
\mu_{2}=b_{1}(\mu) \text { in Case I }
$$

and

$$
\mu_{2}=c_{1}(\mu) \text { in Case II. }
$$

Finally, the third parameter should control the Jacobian $J=\lambda_{1} \lambda_{2} \gamma$ of $f_{\mu}$ at the saddle $O_{\mu}$. Therefore, we put for all cases

$$
\mu_{3}=1-\lambda_{1} \lambda_{2} \gamma
$$

Thus, the family $f_{\mu_{1}, \mu_{2}, \mu_{3}}$ can be considered as a general unfolding of the corresponding non-simple homoclinic tangency under conditions A-D.

We construct the first return maps $T_{k}$ using formulae (2.1) and (2.2). By such a way we obtain a formula for $T_{k}$ in the initial (small) variables $\left(x_{1}, x_{2}, y\right) \in U_{0}$ and parameters $\mu_{1}$ and $\mu_{2}$. Next, we rescale the initial variables and parameters

$$
\left(x_{1}, x_{2}, y\right) \mapsto\left(X_{1}, X_{2}, Y\right),\left(\mu_{1}, \mu_{2}, \mu_{3}\right) \mapsto\left(M_{1}, M_{2}, M_{3}\right)
$$

with asymptotically small (as $k \rightarrow \infty$ ) factors, in such a way that map $T_{k}$ is rewritten, in the rescaled variables and parameters, as some regular three-dimensional quadratic map (the limit form) plus asymptotically small (as $k \rightarrow \infty$ ) terms. Moreover, new coordinates $\left(X_{1}, X_{2}, Y\right)$ and parameters $\left(M_{1}, M_{2}, M_{3}\right)$ can take arbitrary finite values at large $k$. This result is formulated as the following lemma. 
Lemma 1 (RESCALING LEMMA). Let $f_{\mu}$ be the three parameter family under consideration. Then, in the space of the parameters there exist infinitely many regions $\Delta_{k}$ accumulating to $\mu=0$ as $k \rightarrow \infty$, such that the map $T_{k}$ in appropriate rescaled coordinates and parameters is asymptotically $C^{r-1}$-close to one of the following limit maps.

1) In Case I, the limit map is

$$
\bar{X}_{1}=-M_{3} X_{2}, \quad \bar{X}_{2}=Y, \quad \bar{Y}=M_{1}-X_{1}+M_{2} X_{2}-Y^{2},
$$

where

$$
M_{1}=-d \gamma^{2 k}\left(\mu_{1}+\lambda_{1}^{k} c_{1} x_{1}^{+}+\nu_{k}^{1}\right), M_{2}=c_{1}\left(\mu_{2}+\rho_{k}^{1}\right)\left(\lambda_{1} \gamma\right)^{k}, M_{3}=J_{1}\left(\lambda_{1} \lambda_{2} \gamma\right)^{k}
$$

and $\nu_{k}^{1}=O\left(|\gamma|^{-k}+\hat{\lambda}^{k}\right), \rho_{k}^{1}=O\left(\left|\hat{\lambda} / \lambda_{1}\right|^{k}\right)$.

2) In Case II, the limit map is

$$
\bar{X}_{1}=Y, \quad \bar{X}_{2}=X_{1}, \quad \bar{Y}=M_{1}+M_{2} X_{1}+M_{3} X_{2}-Y^{2},
$$

where

$$
\begin{aligned}
& M_{1}=-d \gamma^{2 k}\left[\mu_{1}-\gamma^{-k} y^{-}+\lambda_{1}^{k}\left(\mu_{2} x_{1}^{+}+\nu_{k}^{2}\right)\right], M_{2}=b_{1}\left(\mu_{2}+\rho_{k}^{2}\right)\left(\lambda_{1} \gamma\right)^{k} \\
& M_{3}=J_{1}\left(\lambda_{1} \lambda_{2} \gamma\right)^{k}
\end{aligned}
$$

and $\nu_{k}^{2}, \rho_{k}^{2}=O\left(\left|\hat{\lambda} / \lambda_{1}\right|^{k}+\left|\lambda_{1}\right|^{k}\right)$.

If $M_{3}$ is separated from zero, maps (2.8) and (2.10) are equivalent (map (2.8) takes form (2.10) after scaling $X_{1} \rightarrow-M_{3} X_{1}$ ). Then dynamics of the first return maps for $\mu \in \Delta_{k}$ is the same as for the three-dimensional Hénon map (0.1).

Thus, we need only to proof the Rescaling Lemma.

\section{Proof of Lemma 1.}

Note that under the assumptions $A$ and $B$ we have that $\left|\lambda_{1}\right|>\left|\lambda_{2}\right|,\left|\lambda_{1} \gamma\right|>1$ and $\left|\lambda_{2} \gamma\right|>1$. In principle, the maps $T_{k}$ are rescaled differently in the Cases I and II. However, there is a preparation part of the proof that is conducted in the same way for both the cases. 


\subsection{Preparation form of map $T_{k}$ for rescaling.}

Using (2.2) and (2.1) one can write the map $T_{k}=T_{1} T_{0}^{k}$ for sufficiently large $k$ and small $\mu$ in the form

$$
\begin{aligned}
& \bar{x}_{1}-x_{1}^{+}(\mu)=a_{11}\left(\lambda_{1}^{k} x_{1}+\hat{\lambda}^{k} \xi_{k 1}(x, y, \mu)\right)+a_{12}\left(\lambda_{2}^{k} x_{2}+\hat{\lambda}^{k} \xi_{k 2}(x, y, \mu)\right)+ \\
& +b_{1}\left(y-y^{-}(\mu)\right)+O\left(\left(\hat{\lambda}^{k}+\lambda_{1}^{2 k}\right)\|x\|^{2}+\left|y-y^{-}\right|^{2}\right), \\
& \bar{x}_{2}-x_{2}^{+}(\mu)=a_{12}\left(\lambda_{1}^{k} x_{1}+\hat{\lambda}^{k} \xi_{k 1}(x, y, \mu)\right)+a_{22}\left(\lambda_{2}^{k} x_{2}+\hat{\lambda}^{k} \xi_{k 2}(x, y, \mu)\right)+ \\
& +b_{2}\left(y-y^{-}(\mu)\right)+O\left(\left(\hat{\lambda}^{k}+\lambda_{1}^{2 k}\right)\|x\|^{2}+\left|y-y^{-}\right|^{2}\right), \\
& \gamma^{-k} \bar{y}-\hat{\gamma}^{-k} \eta_{k}(\bar{x}, \bar{y}, \mu)=\mu_{1}+c_{1}\left(\lambda_{1}^{k} x_{1}+\hat{\lambda}^{k} \xi_{k 1}(x, y, \mu)\right)+c_{2}\left(\lambda_{2}^{k} x_{2}+\right. \\
& \left.+\hat{\lambda}^{k} \xi_{k 2}(x, y, \mu)\right)+d\left(y-y^{-}\right)^{2}+O\left(\left(\hat{\lambda}^{k}+\lambda_{1}^{2 k}\right)\|x\|^{2}+\lambda_{1}^{k}\|x\|\left|y-y^{-}\right|+\left|y-y^{-}\right|^{3}\right) .
\end{aligned}
$$

We shift coordinates $x_{1 \text { new }}=x_{1}-x_{1}^{+}(\mu)+\phi_{k}^{1}(\mu), x_{2 n e w}=x_{2}-x_{2}^{+}(\mu)+\phi_{k}^{2}(\mu), y_{\text {new }}=$ $y-y^{-}(\mu)+\psi_{k}(\mu)$, where $\phi_{k}, \psi_{k}=O\left(\left|\lambda_{1}\right|^{k}\right)$, in such a way that the right sides of (3.1) do not contain constant terms for the first two equations and linear in $y_{n e w}$ terms for the third equation. Then (3.1) takes the form

$$
\begin{aligned}
& \bar{x}_{1}=a_{11} \lambda_{1}^{k} x_{1}+b_{1} y+\left(\hat{\lambda}^{k}+\lambda_{1}^{2 k}\right) O(\|x\|)+\lambda_{1}^{k} O(|y|)+O\left(y^{2}\right), \\
& \bar{x}_{2}=a_{21} \lambda_{1}^{k} x_{1}+b_{2} y+\left(\hat{\lambda}^{k}+\lambda_{1}^{2 k}\right) O(\|x\|)+\lambda_{1}^{k} O(|y|)+O\left(y^{2}\right), \\
& \bar{y}-(\hat{\gamma} / \gamma)^{-k} \eta_{k}\left(\bar{x}+x^{+}+\phi_{k}, \bar{y}+y^{-}+\psi_{k}, \mu\right)=M_{k}+d\left(1+s_{k}^{1}\right) \gamma^{k} y^{2}+ \\
& \lambda_{1}^{k} \gamma^{k}\left(c_{1} x_{1}+\left(\left|\lambda_{1}\right|^{k}+\left|\hat{\lambda} / \lambda_{1}\right|^{k}\right) O(\|x\|)\right)+\lambda_{1}^{k} \gamma^{k} O(\|x y\|)+\gamma^{k} O\left(y^{3}\right),
\end{aligned}
$$

where $s_{k}^{1}=O\left(\lambda_{1}^{k}\right)$ and

$$
M_{k}=\gamma^{k}\left[\mu_{1}-\gamma^{-k}\left(y^{-}+\ldots\right)+\lambda_{1}^{k}\left(c_{1} x_{1}^{+}+\ldots\right)\right]
$$

and dots stand for coefficients tending to zero as $k \rightarrow \infty$.

Consider the third equation of (3.2). First of all, we transform its left side. Namely, we write $\bar{y}-(\hat{\gamma} / \gamma)^{-k} \eta_{k}=\bar{y}+(\hat{\gamma} / \gamma)^{-k}\left[\eta_{k}^{0}+\eta_{k}^{1}(\bar{x}, \mu)+\eta_{k}^{2}(\bar{y}, \mu)+\eta_{k}^{3}(\bar{x}, \bar{y}, \mu)\right]$ where $\eta_{k}^{1}(0, \mu)=$ $0, \eta_{k}^{2}(0, \mu)=0$ and $\eta_{k}^{3}=O(\|\bar{x} \bar{y}\|)$. Next, we transfer constant term $(\hat{\gamma} / \gamma)^{-k} \eta_{k}^{0}$ into the right side and join it to $M_{k}$; we substitute $\bar{x}$ from the first two equations of (3.2) into function $\eta_{k}^{1}(\bar{x}, \mu)$ and transfer the obtained function to the right side. After this, all coefficients (in the third equation) get additions of order $O\left(\hat{\gamma}^{-k}\right)$ and a new linear term $p_{k} y=O\left([\hat{\gamma} / \gamma]^{-k}\right) y$

appears. By the shift of coordinates of the form $(x, y) \mapsto(x, y)+O\left([\hat{\gamma} / \gamma]^{-k}\right)$, we vanish both this linear term and constant terms in the right sides of the first and second equations. Note also, that now the left side of the third equation can be written as follows

$$
\begin{aligned}
& \bar{y}+(\hat{\gamma} / \gamma)^{-k} O(\bar{y})+(\hat{\gamma} / \gamma)^{-k} O(\|\bar{x} \bar{y}\|)= \\
& \quad=\bar{y}\left(1+q_{k}\right)+(\hat{\gamma} / \gamma)^{-k} O\left(|\bar{y}|^{2}\right)+(\hat{\gamma} / \gamma)^{-k} O(\|\bar{x} \bar{y}\|)
\end{aligned}
$$


where $q_{k}=O\left([\hat{\gamma} / \gamma]^{-k}\right)$. After this, we can write system (3.2) in the form

$$
\begin{aligned}
\bar{x}_{1} & =a_{11} \lambda_{1}^{k} x_{1}+b_{1} y+\left(\hat{\lambda}^{k}+\lambda_{1}^{2 k}\right) O(\|x\|)+\lambda_{1}^{k} O(|y|)+O\left(y^{2}\right), \\
\bar{x}_{2} & =a_{21} \lambda_{1}^{k} x_{1}+b_{2} y+\left(\hat{\lambda}^{k}+\lambda_{1}^{2 k}\right) O(\|x\|)+\lambda_{1}^{k} O(|y|)+O\left(y^{2}\right), \\
\bar{y}(1 & \left.+q_{k}\right)+(\hat{\gamma} / \gamma)^{-k} O\left(|\bar{y}|^{2}\right)+(\hat{\gamma} / \gamma)^{-k} O(\|\bar{x} \bar{y}\|)=M_{k}+d \gamma^{k}\left(1+s_{k}\right) y^{2}+ \\
& +\lambda_{1}^{k} \gamma^{k}\left[c_{1} x_{1}+\left(\left|\lambda_{1}\right|^{k}+\left|\hat{\lambda} / \lambda_{1}\right|^{k}+\hat{\gamma}^{-k}\right) O(\|x\|)\right]+\lambda_{1}^{k} \gamma^{k} O(\|x y\|)+\gamma^{k} O\left(y^{3}\right),
\end{aligned}
$$

where $s_{k}=O\left(\left|\lambda_{1}\right|^{k}+|\hat{\gamma} / \gamma|^{-k}\right)$ and new $M_{k}$ satisfies (3.3).

\subsection{Proof of item 1 of Lemma 1 .}

In the Case I we have $b_{1}(0)=0, c_{1} \neq 0$ and $b_{2} \neq 0$. We choose $\mu_{1}$ and $\mu_{2} \equiv b_{1}(\mu)$ as the governing parameters. Consider map (3.4) and introduce new coordinates

$$
x_{1 \text { new }}=x_{1}+\frac{1}{c_{1}}\left(\left|\lambda_{1}\right|^{k}+\left|\hat{\lambda} / \lambda_{1}\right|^{k}+\hat{\gamma}^{-k}\right) O(\|x\|), x_{2 n e w}=x_{2}, y_{\text {new }}=y,
$$

i.e. we take as $x_{1 n e w}$ the expression from the square brackets in the third equation of (3.4). Then (3.4) is rewritten in the form

$$
\begin{aligned}
& \bar{x}_{1}=\left(a_{11}+\hat{a}_{k}^{1}\right) \lambda_{1}^{k} x_{1}+\hat{a}_{k} x_{2}+\left(\mu_{2}+\rho_{k}^{1}\right) y+\left(\lambda_{1}^{2 k}+\hat{\lambda}^{k}\right) O\left(\|x\|^{2}\right)+O\left(y^{2}\right), \\
& \bar{x}_{2}= a_{21} \lambda_{1}^{k} x_{1}+\left(b_{2}+\hat{r}_{k}\right) y+\left(\lambda_{1}^{2 k}+\hat{\lambda}^{k}\right) O(\|x\|)+O\left(y^{2}\right) \\
& \bar{y}\left(1+q_{k}\right)+\lambda_{1}^{k} O\left(|\bar{y}|^{2}\right)+\lambda_{1}^{k} O(\|\bar{x} \bar{y}\|)=M_{k}+\lambda_{1}^{k} \gamma^{k} c_{1} x_{1}+d \gamma^{k}\left(1+s_{k}\right) y^{2}+ \\
& \quad+\lambda_{1}^{k} \gamma^{k} O(\|x y\|)+\gamma^{k} O\left(y^{3}\right),
\end{aligned}
$$

where $\rho_{k}^{1}, \hat{r}_{k}=O\left(\lambda_{1}^{k}\right)$ and $\hat{a}_{k}=O\left(\lambda_{1}^{2 k}+\hat{\lambda}^{k}\right)$. Now we rescale the coordinates as follows

$$
y=-\frac{\gamma^{-k}\left(1+q_{k}\right)}{d\left(1+s_{k}\right)} Y, x_{1}=\frac{\gamma^{-k}\left(1+q_{k}\right)}{c_{1} d\left(1+s_{k}\right)\left(\lambda_{1} \gamma\right)^{k}} X_{1}, x_{2}=-\frac{\left(b_{2}+\hat{r}_{k}\right)\left(1+q_{k}\right) \gamma^{-k}}{d\left(1+s_{k}\right)} X_{2} .
$$

Then system (3.5) is rewritten in the new coordinates as follows

$$
\begin{aligned}
& \bar{X}_{1}=-J_{k} X_{2}+M_{2} Y+O\left(\lambda_{1}^{k}\right), \\
& \bar{X}_{2}=Y+O\left(\gamma^{-k}\right), \\
& \bar{Y}+=M_{1}-X_{1}-Y^{2}+O\left(\gamma^{-k}\right),
\end{aligned}
$$

where formula (2.9) is valid for $M_{1}, M_{2}$ and $J_{k}$. Note, that the coefficient $J_{k}$ in (3.6) is nothing other as the Jacobian of map (3.6) in the point $X=0, Y=0$, and, hence, $J_{k}$ coincides, in the main order in $k$, with the Jacobian of map $T_{1} T_{0}^{k}$.

We introduce the new $X_{1}$-coordinate as follows: $X_{1 \text { new }}=X_{1}-M_{2} X_{2}$. After this the rescaled map (3.6) takes the form (2.8) with $M_{3}=J_{k}$. 


\subsection{Proof of item 2 of Lemma 1.}

In the Case II we have $c_{1}(0)=0$ and $b_{1} \neq 0$. We choose $\mu_{1}$ and $\mu_{2} \equiv c_{1}(\mu)$ as the governing parameters. Consider map (3.4) and introduce the new coordinates as $x_{1 \text { new }}=$ $x_{1}, x_{2 \text { new }}=x_{2}-\left(b_{2} / b_{1}\right) x_{1}, y_{\text {new }}=y$. Then (3.4) recasts as

$$
\begin{aligned}
& \bar{x}_{1}=a_{11} \lambda_{1}^{k} x_{1}+b_{1} y+\left(\hat{\lambda}^{k}+\lambda_{1}^{2 k}\right) O(\|x\|)+O\left(y^{2}\right) \\
& \bar{x}_{2}=a_{21}^{\prime} \lambda_{1}^{k} x_{1}+\left(\hat{\lambda}^{k}+\lambda_{1}^{2 k}\right) O(\|x\|)+O\left(y^{2}\right) \\
& \bar{y}\left(1+q_{k}\right)+\lambda_{1}^{k} O\left(|\bar{y}|^{2}\right)+\lambda_{1}^{k} O(\|\bar{x} \bar{y}\|)=M_{k}+d \gamma^{k}\left(1+s_{k}\right) y^{2}+\lambda_{1}^{k} \gamma^{k}\left(\mu_{2}+\rho_{k}^{2}\right) x_{1}+ \\
& \quad+\hat{\lambda}^{k} \gamma^{k} O\left(\left|x_{2}\right|+\|x\|^{2}\right)+\lambda_{1}^{k} \gamma^{k} O(\|x y\|)+\gamma^{k} O\left(y^{3}\right),
\end{aligned}
$$

where $\rho_{k}^{2}=O\left(\left|\lambda_{1}\right|^{k}+\left|\hat{\lambda} / \lambda_{1}\right|^{k}\right), a_{21}^{\prime}=a_{21}-\left(b_{2} / b_{1}\right) a_{11}$ and $M_{k}=\gamma^{k}\left[\mu_{1}-\gamma^{-k} y^{-}+\lambda_{1}^{k} \mu_{2} x_{1}^{+}+\right.$ $\left.O\left(\hat{\lambda}^{k}+\lambda_{1}^{2 k}\right)\right]$ in the case under consideration. Since $c_{1}(0)=0$, condition $J_{1} \neq 0$ (see (2.3)) implies that $a_{21}^{\prime}(0) \neq 0$ and therefore $a_{21}^{\prime}(\mu) \neq 0$ for small $\mu$. Now we rescale the coordinates as follows

$$
y=-\frac{\gamma^{-k}\left(1+q_{k}\right)}{d\left(1+s_{k}\right)} Y, x_{1}=-\frac{b_{1} \gamma^{-k}\left(1+q_{k}\right)}{d\left(1+s_{k}\right)} X_{1}, x_{2}=-\lambda_{1}^{k} \gamma^{-k} \frac{b_{1} a_{21}^{\prime}\left(1+q_{k}\right)}{d\left(1+s_{k}\right)} X_{2} .
$$

After this, we can rewrite (3.7) in the following form

$$
\begin{aligned}
& \bar{X}_{1}=Y+O\left(\lambda_{1}^{k}\right), \\
& \bar{X}_{2}=X_{1}+O\left(\left(\lambda_{1} \gamma\right)^{-k}\right), \\
& \bar{Y}=M_{1}+M_{2} X_{1}+J_{k} X_{2}-Y^{2}+O\left(\lambda_{1}^{k}\right) .
\end{aligned}
$$

where formula (2.11) is valid for $M_{1}, M_{2}$ and $J_{k}$. It completes the proof.

\section{Aknowledgements}

The paper was supported by grant 14-41-00044 of the RSF. The first and the second authors were partially supported by grants of RFBR No.13-01-00589, 13-01-97028-povolzhje and 14-01-00344, the second author was also supported by the Leverhulme Trust grant RPG-279 and the EPSRC Mathematics Platform grant EP/I019111/1, and the third author was supported by the MEC grant MTM2009-09723 (Spain) and the CIRIT grant 2009 SGR 67 (Spain).

\section{References}

[1] S.V. Gonchenko, J.D. Meiss, I.I. Ovsyannikov. Chaotic dynamics of three-dimensional Hénon maps that originate from a homoclinic bifurcation. Regul. Chaotic Dyn., 2006, vol. 11, pp. 191-212. 
[2] D.V. Turaev, L.P. Shilnikov. Pseudo-hyperbolisity and the problem on periodic perturbations of Lorenz-like attractors. Russian Dokl. Math., 2008, vol. 467, pp. 23-27. English transl.: Doklady Mathematics, 2008, vol. 77, no. 1, pp. 17-22.

[3] Gonchenko S.V., Ovsyannikov I.I., Simó C. and Turaev D., Three-dimensional Hénon-like maps and wild Lorenz-like attractors. Int. J. of Bifurc. and Chaos, 2005, vol. 15, pp. 3493-3508.

[4] A.L. Shilnikov, L.P. Shilnikov, D.V. Turaev. Normal forms and Lorenz attractors. Int. J. of Bifurc. and Chaos, 1993, vol. 3, pp. 1123-1139.

[5] A.L. Shilnikov. Bifurcation and chaos in the Morioka-Shimizu system. Methods of qualitative theory of differential equations, Gorky, 1986, pp. 180-193. English translation Selecta Math. Soviet., 1991, vol. 10, pp. 105-117]; II. Methods of Qualitative Theory and Theory of Bifurcations, Gorky, 1989, pp. 130-138.

[6] A.L. Shilnikov. On bifurcations of the Lorenz attractor in the Shimuizu-Morioka model. Physica D, 1993, vol. 62, pp. 338-346.

[7] S.V. Gonchenko, A.S. Gonchenko, I.I. Ovsyannikov, D.V. Turaev Examples of Lorenz-like attractors in Hénon-like maps, Mat. Model. of Nat. Phenom, 2013, vol. 8, no. 5, pp. 48-70.

[8] A.S. Gonchenko. On Lorenz-like attractors in model of a Celtic stone. Vestnik UdSU, Math., Mech. and Comp. Sci., 2013, vol. 2, pp. 3-11.

[9] A.S. Gonchenko, S.V. Gonchenko. On the existence of Lorenz-like attractors in the nonholonomic model of a "Celtic stone". Rus. J. of Nonlin. Dyn., 2013, vol. 9, no. 1, pp. $77-89$.

[10] A.S. Gonchenko, S.V. Gonchenko, A.O. Kazakov. Richness of Chaotic Dynamics in Nonholonomic Models of a Celtic Stone. Regular and Chaotic Dynamics, 2013, vol. 18, no. 5, pp. 521-538.

[11] A.S. Gonchenko, S.V. Gonchenko, L.P. Shilnikov. Towards scenarios of chaos appearance in three-dimensional maps. Rus. J. Nonlinear Dynamics, 2012, vol. 8, pp. $3-28$.

[12] Gonchenko A.S., Gonchenko S.V., Kazakov A.O., Turaev D.V. The simplest scenarios of onset of chaos in three-dimensional maps. Int. J. of Bifurc. and Chaos, 2005, vol. 15, pp. 3493-3508. 
[13] D.V.Turaev, L.P.Shilnikov. An example of a wild strange attractor. Sb. Math, 1998, vol. 189, no. 2, pp. 137-160.

[14] S.E. Newhouse. The abundance of wild hyperbolic sets and non-smooth stable sets for diffeomorphisms. IHES Publ. Math., 1979, vol. 50, pp. 101-151.

[15] V.S. Aframovich, L.P. Shilnikov. Strange attractors and quasiattractors. Nonlinear Dynamics and Turbulence, eds. G.I.Barenblatt, G.Iooss, D.D.Joseph, Boston, Pitmen, 1983.

[16] S.V.Gonchenko, L.P.Shilnikov, D.V.Turaev. Dynamical phenomena in systems with structurally unstable Poincare homoclinic orbits. Russian Acad. Sci. Dokl. Math, 1993, vol. 47, no. 3, pp. 410-415.

[17] S.V.Gonchenko, L.Shilnikov, D.Turaev. On global bifurcations in three-dimensional diffeomorphisms leading to wild Lorenz-like attractors. Regul. and Chaotic Dyn., 2009, vol. 14, pp. 137-147.

[18] S.V. Gonchenko, I.I. Ovsyannikov. On bifurcations of three-dimensional diffeomorphisms with a non-transversal heteroclinic cycle containing saddle-foci. Rus. J. Nonlinear Dynamics, 2010, vol. 6, pp. 61-77.

[19] S.V. Gonchenko, I.I. Ovsyannikov. On Global Bifurcations of Three-dimensional Diffeomorphisms Leading to Lorenz-like Attractors. Mat. Model. of Nat. Phenom, 2013, vol. 8, no. 5, pp. 71-83.

[20] D.V.Turaev. On dimension of nonlocal bifurcational problems. Int. J. of Bifurcation and Chaos, 1996, vol. 6, no. 5, pp. 919-948.

[21] J.C.Tatjer. Three-dimensional dissipative diffeomorphisms with homoclinic tangencies. Ergod.Th. and Dynam. Sys., 2001, vol. 21, pp. 249-302.

[22] Newhouse S.E., Palis J., Takens F. Bifurcations and stability of families of diffeomorphisms. Publ. Math. Inst. Haute Etudes Scientifiques, 1983, iss. 57, pp. 5-71.

[23] S.V.Gonchenko, V.S.Gonchenko, J.C.Tatjer. Bifurcations of three-dimensional diffeomorphisms with non-simple quadratic homoclinic tangencies and generalized Henon maps. Regular and Chaotic Dynamics, 2007, vol. 12, no. 3, pp. 233-266.

[24] S.V.Gonchenko, L.P.Shilnikov, D.V.Turaev. Dynamical phenomena in systems with structurally unstable Poincare homoclinic orbits. Interdisc. J. CHAOS, 1996, vol. 6, no. 1 , pp. $15-31$. 
[25] S.V.Gonchenko, L.P.Shilnikov, D.V.Turaev. On dynamical properties of multidimensional diffeomorphisms from Newhouse regions. Nonlinearity, 2008, vol. 21, pp. 923972.

[26] C.Mira, L.Gardini, A.Barugola, J.C.Cathala. Chaotic dynamics in two-dimensional noninvertible maps. World Scientific, Singapore. 1996.

[27] A.Pumariño, J.C.Tatjer. Dynamics near homoclinic bifurcations of three-dimensional dissipative diffeomorphisms. Nonlinearity, 2006, vol. 19, pp. 2833-2852.

[28] A.Pumariño, J.C.Tatjer. Attractors for return maps near homoclinic tangencies of three-dimensional dissipative diffeomorphisms. Discrete and Continuous Dynamical Systems, series B, 2007, vol. 8, no. 4, pp. 971-1006.

[29] A. Arneodo, P. Coullet, C. Tresser. Possible new strange attractors with a spiral structure. Commun. Math. Phys., 1981, vol. 79, iss. 4, pp. 573-579.

[30] L.P.Shilnikov, A.L.Shilnikov, D.V.Turaev and L.O.Chua. Methods of Qualitative Theory in Nonlinear Dynamics, Part I. World Scientific. 1998.

[31] S.V.Gonchenko, L.P.Shilnikov. Invariants of $\Omega$-conjugacy of diffeomorphisms with a nontransversal homoclinic orbit. Ukr. Math. J, 1990, vol. 42, no. 2, pp. 134-140.

[32] S.V.Gonchenko, L.P.Shilnikov. On moduli of systems with a nontransversal Poincare homoclinic orbit. Russian Acad. Sci. Izv. Math, 1993, vol. 41, no. 3, pp. 417-445.

[33] M.W.Hirsch, C.C.Pugh, M.Shub. Invariant manifolds. Lecture Notes in Math, 1977, vol. 583, Springer-Verlag, Berlin. 